\title{
$\mathrm{Cl}$ i ni copat hol ogi cal si gni f i cance of ser um fractal ki ne in pri mary bi I i ary ci rrhosi s
}

\begin{tabular}{|l|l|}
\hline 著者 & $\begin{array}{l}\text { Har ada Keni chi, Kakuda Yuko, Nakamur a M nor u, } \\
\text { Shi noda Shi nj i, Nakanuma Yasuni }\end{array}$ \\
\hline $\begin{array}{l}\text { j our nal or } \\
\text { publ i cat i on t i t l e }\end{array}$ & Di gest i ve Di seases and Sci ences \\
\hline vol une & 58 \\
\hline number & 10 \\
\hline page r ange & $3037-3043$ \\
\hline year & $2013-10-01$ \\
\hline URL & ht t p: //hdl . handl e. net /2297/36278 \\
\hline
\end{tabular}




\section{Clinicopathological significance of serum fractalkine in primary biliary cirrhosis}

Kenichi Harada ${ }^{1)}$, Yuko Kakuda ${ }^{1)}$, Minoru Nakamura ${ }^{2)}$, Shinji Shimoda ${ }^{3)}$, Yasuni Nakanuma ${ }^{1)}$

1) Department of Human Pathology, Kanazawa University Graduate School of Medicine, Kanazawa, Japan

2) Clinical Research Center in National Hospital Organization (NHO) Nagasaki Medical Center and Department of Hepatology, Nagasaki University Graduate School of Biomedical Sciences, Omura, Japan

3) Department of Medicine and Biosystemic Science, Graduate School of Medical Sciences, Kyushu University, Fukuoka, Japan

Short title: Fractalkine in PBC

\section{Number of figures: 4}

List of abbreviations: PBC, Primary biliary cirrhosis; UDCA, ursodeoxycholic acid; ADAM, a disintegrin and metalloprotease; RA, rheumatoid arthritis; IBD, inflammatory bowel disease; CNSDC, chronic nonsuppurative destructive cholangitis; TLR, Toll-like receptor; AMA, anti-mitochondrial antibody; ULN, upper limit of normal

A conflict of interest statement: The authors report no conflicts of interest.

Financial support: This work was supported by grant No. 23590393 from the Ministry of Education, Culture, Sports, Science and Technology of Japan (K.H.) and by Health and Labour Sciences Research Grants for the Research on Measures for Intractable Diseases (Chief Tsubouchi, M.D.).

Address correspondence to:

Kenichi Harada, M.D.

Department of Human Pathology

Kanazawa University Graduate School of Medicine

Kanazawa 920-8640, Japan

FAX: (0)76-234-4229 (Japan)

TEL: (0)76-265-2199 (Japan)

E-mail: kenichih@med.kanazawa-u.ac.jp 


\begin{abstract}
Background: Primary biliary cirrhosis (PBC), characterized by cholangitis and loss of intrahepatic small bile ducts, predominantly affects middle-aged females. We have reported that fractalkine expression associated with chronic inflammation is observed in the damaged bile ducts and periductal vessels of PBC patients, which is closely associated with chronic cholangitis. Aims: We investigated the association between serum fractalkine levels and clinicopathological findings in PBC patients. Methods: Liver biopsy specimens before ursodeoxycholic acid (UDCA) treatment and serum samples at the time of liver biopsy and 1 and 2 years after treatment were obtained from 68 PBC patients $(\mathrm{M} / \mathrm{F}=14 / 54)$. Serum fractalkine levels were measured by enzyme-linked immunosorbent assay (ELISA), and their association with clinicopathological findings (liver function data, autoantibodies, cholangitis activity, hepatitis activity, fibrosis, bile duct loss, and orcein-positive granules) was analyzed. Results: Serum fractalkine levels were in the range of 0.1-33.2 ng/ml (average, $3.2 \mathrm{ng} / \mathrm{ml}$ ). They were increased in PBC patients with high degrees of cholangitis activity, a mild degree of hepatitis activity, fibrosis, orcein-positive granules, and early stages. In cases with high serum fractalkine levels, those who exhibited good biochemical responses to treatment mostly showed improved serum fractalkine levels after treatment. Conclusion: Serum fractalkine levels of PBC patients were high in cases with marked cholangitis activity at early stages. In addition, they closely correlated with the effect of therapy, indicating that fractalkine plays a role in the pathogenesis of initial cholangitis in early stage PBC and consequent chronic cholangitis. Thus, our results suggest that fractalkine is a good candidate for molecular-targeted treatment.
\end{abstract}

Key Words: primary biliary cirrhosis, gp210, fractalkine, pathology, cholangitis 


\section{INTRODUCTION}

Fractalkine (CX3CL1) plays an important role in leukocytes migration to target sites under both physiological and pathological conditions. Unlike other chemokines, fractalkine is a membrane-bound protein that can be shed in soluble chemotactic form following cleavage by a disintegrin and metalloprotease (ADAM) 10 and ADAM17 [1]. Soluble fractalkine is known to be a potent chemoattractant for CD8-positive and CD4-positive T cells, CD16-positive natural killer cells, and macrophage/monocytes expressing its receptor (CX3CR1), and promotes strong adhesion of these leukocytes in an integrin-independent manner. Therefore, fractalkine signaling is thought to be involved in the development of chronic inflammation, as has been reported in cases of rheumatoid arthritis (RA), atherosclerosis, inflammatory bowel disease (IBD), and rejection of implanted organs, and genetic polymorphisms of fractalkine have been speculated to increase disease susceptibility [2]. Moreover, fractalkine was recently noted as a molecular target of therapeutic agents for RA and IBD, and anti-inflammatory treatments using anti-CX3CR1 antibody have been developed for RA and an animal model of heart transplantation [2,3].

Primary biliary cirrhosis (PBC) mainly affects middle-aged females; histologically, the interlobular bile ducts are primarily damaged with characteristic findings such as chronic nonsuppurative destructive cholangitis (CNSDC) followed by progressive loss of bile ducts [4]. There is considerable evidence that bile duct damage is mediated by autoreactive or cytotoxic T cells [5-8], and the molecular mechanisms responsible for the migration of pathogenic $\mathrm{T}$ cells around or within bile ducts have been clarified over the past several years. We previously reported that the level of fractalkine is significantly elevated in the sera of PBC patients and in small bile ducts, particularly those that are damaged. In addition, vascular endothelial cells expressing fractalkine is increased in PBC [9], suggesting that fractalkine is an important mediator associated with the continuous portal, particularly periductal, inflammation of PBC. Moreover, the expression of fractalkine in bile ducts is reported to be associated with innate immunity via Toll-like receptor (TLR) 3 and TLR4 in vascular endothelial cells, infiltrating mononuclear cells, and biliary epithelial 
cells $[10,11]$.

Fractalkine is an important chemokine closely associated with the pathogenesis of cholangiopathy, and is likely involved in the continuous inflammation of chronic cholangitis in PBC. In this study, we investigated serum fractalkine levels in PBC patients and their association with clinicopathological findings.

\section{MATERIALS and METHODS}

Subjects and clinical information: Sixty-eight patients with PBC were selected from registered files of the National Hospital Organization (NHO) Nagasaki Medical Center. The patients included 14 males and 54 females with average ages of 53 and 59 years, respectively. Serum samples were obtained at the diagnosis of PBC or before ursodeoxycholic acid (UDCA) treatment for PBC and 1 and 2 years after starting UDCA treatment, and liver function data [aspartate transaminase (AST), alkaline phosphatase (ALP) levels], IgM levels, and levels of autoantibodies [anti-mitochondrial antibody (AMA), anti-centromere antibody, and anti-gp210 antibody] at were analyzed at each time point. The reserved serum samples were used for the measurement of soluble fractalkine with an enzyme-linked immunosorbent assay (ELISA) kit (R\&D Systems, Minneapolis, MN). Liver biopsy specimens from all cases were also obtained at the time PBC was diagnosed, before treatment.

Diagnosis of PBC and criteria of UDCA effect: The diagnosis of PBC and biochemical response to UDCA were defined following criteria established by the Intractable Hepato-Biliary Disease Study Group in Japan. Patients whose condition meets one of the below criteria were diagnosed with PBC. (1) Histologically observed chronic nonsuppurative destructive cholangitis (CNSDC) and laboratory findings not contradicting PBC; (2) positive AMA and/or anti-pyruvate dehydrogenase (PDH) antibody, CNSDC not histologically observed but histological findings compatible with PBC; or (3) histological examination not performed, but positive AMA or anti-PDH antibodies and clinical findings and course indicating PBC. Biochemical response to UDCA was as follows: good, normalization of serum ALP, ALT, and IgM within 2 years of starting UDCA 
treatment; fair, serum ALP, ALT, and IgM within $<1.5$ upper limit of normal (ULN) 2 years after starting UDCA treatment; and poor, serum ALP, ALT, and IgM within $\geq 1.5$ ULN 2 years after starting UDCA treatment.

Histological examination: More than $104-\mu$ m-thick sections were prepared from each paraffin-embedded block; several were stained with hematoxylin-eosin (HE), Gomori’s reticulum, and Orcein stain for histological diagnosis, grading, and staging.

The grading system and new staging system proposed by Nakanuma [12] were used to evaluate disease activity and stage. In summary, chronic cholangitis activity (CA) was categorized into four grades (CA0-3) according to the degree and distribution. CA0 (no activity) was defined as absent or ambiguous bile duct damage. In CA1 (mild activity), one bile duct showed evident chronic cholangitis. In CA2 (moderate activity), two or more bile ducts were affected. In CA3 (marked activity), at least one damaged bile duct showed CNSDC and/or granulomatous cholangitis. Evident chronic cholangitis was defined as a damaged bile duct entirely surrounded by mild to moderate, duct-oriented lymphoplasmacytic inflammation. Hepatitis activity (HA) was also categorized into four grades (HA0-3) according to the presence and degree of interface hepatitis and lobular hepatitis. In HA0 (no activity), interface hepatitis was not present. The presence of interface hepatitis affecting at least 10 continuous hepatocytes at the interface of one portal tract or fibrous septum was categorized as HA1 (mild activity) and in two or more portal tracts or fibrous septa as HA2 (moderate activity). In HA3 (marked activity), interface hepatitis affecting at least 20 continuous hepatocytes at the limiting plate in more than half of the portal tracts or fibrous septa was present throughout the specimen, with entrapment of hepatocytes in the expanded portal tracts. Although no or minimum lobular hepatitis was found in HA0, mild to moderate lobular hepatitis was observed in HA1 and HA2, and moderate lobular hepatitis in HA3. Occasional zonal necrosis and bridging necrosis was regarded as HA3.

Three factors were evaluated for the new staging system: fibrosis, bile duct loss, and Orcein-positive granule deposition. These three items were scored as follows. For fibrosis, a score of 0 indicated almost no fibrosis or fibrosis limited to the portal tracts, a score of 1 indicated fibrosis 
extending beyond the portal area with occasional incomplete septal fibrosis, a score of 2 indicated completely connecting septal fibrosis or bridging fibrosis with variable lobular distortion, and a score of 3 was assigned for cirrhosis (extensive fibrosis with regenerative nodules). For bile duct loss, interlobular bile ducts were evaluated in well-formed portal tracts with evident hepatic arterial branches and portal vein branches. A score of 0 meant interlobular bile ducts were distinguishable in all portal tracts in specimens. Score of 1 and 2 meant that bile duct loss was evident in $<1 / 3$ and in $1 / 3-2 / 3$ of portal tracts, respectively. A score of 3 indicated that bile ducts were absent in $>2 / 3$ of portal tracts. For Orcein-positive granule deposition, a score of 0 meant no deposition in periportal hepatocytes, a score of 1 indicated deposition in some periportal hepatocytes in $<1 / 3$ of portal tracts, and a score of 3 was given for patients with deposition in many hepatocytes of $>2 / 3$ portal tracts or fibrous septa. Samples intermediate between 1 and 3 were assigned a score of 2 . After each of these items was scored, they were summed: a total score of 0 indicated stage 1 (no or minimum progression), 1-3, stage 2 (mild progression); 4-6, stage 3 (moderate progression); and 7-9, stage 4 (advanced progression).

Statistical analysis: Data were analyzed using Welch’s t-tests, paired t tests, and Spearman rank correlation coefficient; $\mathrm{p}<0.05$ was considered statistically significant for all analyses.

\section{RESULTS}

Serum fractalkine levels and their relationship with serological data: Serum fractalkine levels in PBC patients ranged from 0 to $33.2 \mathrm{ng} / \mathrm{ml}$ before UDCA treatment, and the mean was 3.2 ng/ml. However, most values were 0-3.0 ng/ml, and 14 cases showed high levels ( $>5 \mathrm{ng} / \mathrm{ml}$ ) (Fig. 1). This trend confirmed the findings in our previous report [9], where serum fractalkine levels in all controls including healthy controls and patients with extrahepatic biliary obstruction and HCV-related chronic hepatitis were $<3.0 \mathrm{ng} / \mathrm{ml}$ [9]. Next, the correlation between fractalkine levels and liver function data (ALT and ALP levels) and IgM levels was examined. The correlation 
coefficients between fractalkine levels and ALT, ALP, and IgM levels were 0.12, 0.06, and 0.10, respectively (Fig. 2A). With regard to autoantibodies, none showed significant positive correlation with serum fractalkine levels (Fig. 2B). However, patients with high gp210 titers had low fractalkine levels, whereas patients with high serum fractalkine levels had low gp210 titers, except one case in which both levels were high (fractalkine, $33.2 \mathrm{ng} / \mathrm{ml}$; gp210, 132.4 times) (Fig. 2B). This patient (Fig. 2B, \#) was a 35-year-old male positive for both AMA and ANA and had increased IgG and IgM levels. His condition rapidly deteriorated despite all treatment and he promptly underwent liver transplantation, suggesting the existence of other factors such as autoimmune hepatitis exacerbating liver injury. Therefore, we considered this case as an outlier and performed the subsequent statistical analysis excluding it. Consequently, the serum fractalkine level of $1.5 \pm 0.2 \mathrm{ng} / \mathrm{ml}$ (mean \pm standard error of mean) in patients with high gp210 titers ( $>5$ times) was significantly lower ( $5.5 \pm 1.5 \mathrm{ng} / \mathrm{ml}$ ) than that in patients with low gp210 titers ( $\leq 5$ times) (Welch’s t-test, $\mathrm{p}<0.05$ ). Moreover, gp210 titers (1.7 \pm 0.3 times) in patients with high serum fractalkine levels ( $>3 \mathrm{ng} / \mathrm{ml})$ were significantly lower (35.1 \pm 11.1 times) than those in patients with low fractalkine levels $(<3 \mathrm{ng} / \mathrm{ml})$ (Welch’s t-test, $\mathrm{p}<0.05)$

\section{Correlation between serum fractalkine levels and histological activity and staging of}

PBC: Fractalkine levels in PBC patients were analyzed according to the histological activity of chronic cholangitis (CA) and hepatitic change (HA). As shown in Fig. 3, fractalkine levels in CA3 cases were significantly higher than those in cases with other scores (CA0-CA2) and, in contrast, the fractalkine levels in HA3 cases were significantly lower than those in cases with other scores (HA0-HA2) (Welch's t-test, $\mathrm{p}<0.05$ ) with the exception of one case (Figs. 3A and 3B). As for the three histological findings defining histological stage, fibrosis, bile duct loss, and Orcein-positive granules, the cases with low scores (0-1) of fibrosis and a score of 0 for Orcein-positive granules showed significantly higher fractalkine levels than in cases with a score of 2-3 for fibrosis and 1-3 for Orcein-positive granules (Figs. 3D and 3F) (Welch's t-test, $\mathrm{p}<0.05$ ). Moreover, the cases with 
early histological stages (stages 1-2) showed higher fractalkine levels than those with advanced stages (stages 3-4) (Fig. 3C).

Correlation between serum fractalkine levels and UDCA effects: The changes in serum fractalkine levels before (before treatment) and 1-2 years after the beginning of UDCA treatment (after treatment) are shown in Fig. 4. Most cases with low fractalkine levels ( $<3.0 \mathrm{ng} / \mathrm{ml})$ before the treatment retained low levels. However, 14 cases with high fractalkine levels ( $>5 \mathrm{ng} / \mathrm{ml}$ ) showed changes over time. Among serum ALP, ALT, and IgM defining the biochemical response to UDCA, the patients with $>2$ items of good response or $<1$ item of good response were evaluated separately. The patients with high fractalkine levels $(>5 \mathrm{ng} / \mathrm{ml}$ ) before treatment showed a decrease after treatment in the patients with $>2$ items of good response, but those with high fractalkine levels showed an increase in those with $<1$ item of good response. Statistical analysis demonstrated that in patients with $>2$ good response items, fractalkine level was significantly decreased after treatment $(2.9 \pm 1.1 \mathrm{ng} / \mathrm{ml})$ compared with before treatment $(4.6 \pm 1.6 \mathrm{ng} / \mathrm{ml})$ (paired t test, $\mathrm{p}<0.05)$.

\section{DISCUSSION}

Fractalkine chemoattracts CXCR1-expressing cells in target organs and maintains continuous inflammation, ultimately inducing chronic inflammation. We previously reported that damaged bile ducts and vessels in PBC express fractalkine and that the induction of fractalkine expression in biliary epithelial cells is closely associated with biliary and periductal innate immunity [9,10]. In the present study, we measured serum fractalkine levels using different PBC patients' sera and obtained a similar average and distribution. Most cases had levels $<3 \mathrm{ng} / \mathrm{ml}$, but 14 patients had high levels ( $>5 \mathrm{ng} / \mathrm{ml}$ ). These high levels were only observed in PBC patients; levels in the healthy population and patients with other liver diseases were $<3 \mathrm{ng} / \mathrm{ml}$ [9]. Therefore, in PBC patients with high fractalkine levels, intrahepatic fractalkine produced by liver constituent cells, including biliary epithelial cells, is the likely cause of the increase in serum fractalkine. This indicates that 
fractalkine-mediated inflammation is associated with chronic cholangitis and portal inflammation.

Although a serological hallmark of PBC is the presence of AMA, which is found in $>90 \%$ of patients, ANAs are also detected in PBC patients [13-17]. Anti-gp210 and anti-sp100 antibodies are highly specific for PBC and useful for its diagnosis, particularly those who are negative for AMAs [13-17]. In addition, although AMAs are not associated with disease progression, ANAs are associated with disease severity and are therefore useful as a marker of poor prognosis [15-18]. Anti-gp210 antibodies are the strongest predictive factor among ANAs for progression to end-stage hepatic failure (i.e., hepatic failure type progression) [15-17]. Moreover, the presence of gp210 antibodies is a risk factor for more severe interface hepatitis and lobular inflammation defining hepatitis activity (HA). The present study revealed that most patients with high gp210 titers had low serum fractalkine levels, whereas most patients with high fractalkine levels had low gp210 titers. Moreover, the fractalkine levels in the CA3 cases were significantly higher than those with lower scores (CA0-CA2). In contrast, fractalkine levels in the HA3 cases were significantly lower than those with low scores (HA0-HA2). Therefore, a possible explanation of why high anti-gp210 antibody and fractalkine levels showed opposite trends with each other except in one unique case (Fig. 2B, \#) is that the gp210 antibody and fractalkine are associated with marked hepatitic change (HA) and chronic cholangitis (CA), respectively, indicating that they may reflect two different pathogenetic mechanisms of PBC from the aspects of hepatitis and cholangitis, respectively. The unique case showing high gp210 antibody titers and fractalkine levels (Fig. 2B, \#) had extensive chronic cholangitis (CA2) and hepatitic changes (HA3), as shown in Fig. 3. This PBC case was very rare and pathognomonic, and the patient rapidly progressed to Stage 3 liver failure before the terminal stage without any improvement achieved with UDCA treatment. This indicates that the case was aggressive and had a poor prognosis, suggesting a case of atypical PBC.

As discussed above, fractalkine is speculated to be associated with the pathogenesis of chronic cholangitis in PBC, which is a characteristic feature of this disease. Moreover, the present study revealed that the cases with low fibrosis scores (0-1) and a score of 0 for Orcein-positive granules among the three histological findings defining histological stage showed high fractalkine levels, 
similar to patients in early histological stages (stages $1-2$ ). Therefore, fractalkine plays a role in the pathogenesis of chronic cholangitis in PBC, particularly in the early stages, indicating that fractalkine is an important factor in initial chronic cholangitis in PBC and also during the transition to chronic cholangiopathy in PBC without reference to hepatitic change. However, many PBC patients had low serum fractalkine levels, even in the early stages, indicating that the association of fractalkine in the pathogenesis of PBC might be varied in each case, and its significance differs between early and advanced stages, even in the same patient.

The present study demonstrated a correlation between serum fractalkine levels and response to UDCA; although patients with low fractalkine levels $(<3 \mathrm{ng} / \mathrm{ml})$ before UDCA treatment had low levels after treatment irrespective of the effectiveness of UDCA treatment, the cases with high levels (>5 ng/ml) showed a decrease or increase after UDCA treatment in good and poor biochemical responders, respectively. UDCA is a hydrophilic, nontoxic bile acid that contributes nearly $3 \%$ to the normal bile acid pool in humans and has cytoprotective, anti-apoptotic, membrane stabilizing, antioxidative, and immunomodulatory effects [19]. In our previous study, we demonstrated that upregulation of fractalkine expression in biliary epithelial cells is induced by the biliary innate immune response [9]. Therefore, it is possible that UDCA directly regulates the production of fractalkine in liver constituent cells, including biliary epithelial cells, but further study is needed to clarify the direct correlation between the effectiveness of UDCA and serum fractalkine levels.

The present study demonstrated that among PBC patients, those with high serum fractalkine levels are characterized by low gp210 titers, extensive chronic cholangitis, limited hepatitic change, and early-stage PBC. Therefore, fractalkine is speculated to play a role in the initial pathogenesis of chronic inflammation in early stage PBC and consequently chronic cholangitis. In addition to PBC, fractalkine is associated with chronic inflammation in other diseases, including RA and IBD, and an anti-fractalkine monoclonal antibody has been developed as a clinical molecular treatment. Collectively, our findings suggest that fractalkine-CX3CR1 signaling might be a useful molecular target for the treatment of PBC, particularly in UDCA-ineffective PBC cases with high serum fractalkine levels. 
Harada - $11-$

\section{ACKNOWLEDGEMENTS}

The authors would like to thank Enago (www.enago.jp) for performing an English language review. 


\section{REFERENCES}

1. Bourd-Boittin K, Basset L, Bonnier D, L'Helgoualc'h A, Samson M, Theret $\mathrm{N}$. CX3CL1/fractalkine shedding by human hepatic stellate cells: contribution to chronic inflammation in the liver. J Cell Mol Med, 2009; 13: 1526-1535.

2. Jones BA, Beamer M, Ahmed S. Fractalkine/CX3CL1: a potential new target for inflammatory diseases. Mol Interv, 2010; 10: 263-270.

3. Nishimura M, Kuboi Y, Muramoto K, Kawano T, Imai T. Chemokines as novel therapeutic targets for inflammatory bowel disease. Ann N Y Acad Sci, 2009; 1173: 350-356.

4. Nakanuma Y, Ohta G. Histometric and serial section observations of the intrahepatic bile ducts in primary biliary cirrhosis. Gastroenterology, 1979; 76: 1326-1332.

5. Shimoda S, Ishikawa F, Kamihira T, et al. Autoreactive T-cell responses in primary biliary cirrhosis are proinflammatory whereas those of controls are regulatory. Gastroenterology, 2006; 131: 606-618.

6. Shimoda S, Miyakawa H, Nakamura M, et al. CD4 T-cell autoreactivity to the mitochondrial autoantigen PDC-E2 in AMA-negative primary biliary cirrhosis. J Autoimmun, 2008; 31: $110-115$.

7. Shimoda S, Nakamura M, Ishibashi H, Hayashida K, Niho Y. HLA DRB4 0101-restricted immunodominant $\mathrm{T}$ cell autoepitope of pyruvate dehydrogenase complex in primary biliary cirrhosis: evidence of molecular mimicry in human autoimmune diseases. J Exp Med, 1995; 181: 1835-1845.

8. Shimoda S, Nakamura M, Ishibashi H, et al. Molecular mimicry of mitochondrial and nuclear autoantigens in primary biliary cirrhosis. Gastroenterology, 2003; 124: 1915-1925.

9. Isse K, Harada K, Zen Y, et al. Fractalkine and CX3CR1 are involved in the recruitment of intraepithelial lymphocytes of intrahepatic bile ducts. Hepatology, 2005; 41: 506-516.

10. Shimoda S, Harada K, Niiro H, et al. Interaction between Toll-like receptors and natural killer cells in the destruction of bile ducts in primary biliary cirrhosis. Hepatology, 2011; 53: 
$1270-1281$.

11. Harada K, Ohira S, Isse K, et al. Lipopolysaccharide activates nuclear factor-kappaB through toll-like receptors and related molecules in cultured biliary epithelial cells. Lab Invest, 2003; 83: $1657-1667$.

12. Nakanuma Y, Zen Y, Harada K, et al. Application of a new histological staging and grading system for primary biliary cirrhosis to liver biopsy specimens: Interobserver agreement. Pathol Int, 2010; 60: 167-174.

13. Rigopoulou EI, Davies ET, Pares A, et al. Prevalence and clinical significance of isotype specific antinuclear antibodies in primary biliary cirrhosis. Gut, 2005; 54: 528-532.

14. Invernizzi P, Selmi C, Ranftler C, Podda M, Wesierska-Gadek J. Antinuclear antibodies in primary biliary cirrhosis. Semin Liver Dis, 2005; 25: 298-310.

15. Nakamura M, Kondo H, Mori T, et al. Anti-gp210 and anti-centromere antibodies are different risk factors for the progression of primary biliary cirrhosis. Hepatology, 2007; 45: 118-127.

16. Nakamura M, Komori A, Ito $\mathrm{M}$, et al. Predictive role of anti-gp210 and anticentromere antibodies in long-term outcome of primary biliary cirrhosis. Hepatol Res, 2007; 37 Suppl 3: S412-419.

17. Nakamura M, Shimizu-Yoshida Y, Takii Y, et al. Antibody titer to gp210-C terminal peptide as a clinical parameter for monitoring primary biliary cirrhosis. J Hepatol, 2005; 42: 386-392.

18. Itoh S, Ichida T, Yoshida T, et al. Autoantibodies against a $210 \mathrm{kDa}$ glycoprotein of the nuclear pore complex as a prognostic marker in patients with primary biliary cirrhosis. $J$ Gastroenterol Hepatol, 1998; 13: 257-265.

19. Kumar D, Tandon RK. Use of ursodeoxycholic acid in liver diseases. J Gastroenterol Hepatol, 2001; 16: 3-14. 


\section{FIGURE LEGENDS}

Fig. 1 Distribution of serum fractalkine levels in PBC patients before UDCA treatment. Levels ranged from 0 to $33.2 \mathrm{ng} / \mathrm{ml}$, and the mean was $3.2 \mathrm{ng} / \mathrm{ml}$. Fourteen cases showed high fractalkine levels ( $>5 \mathrm{ng} / \mathrm{ml})$.

Fig. 2A: Correlation between serum fractalkine levels and liver function data (ALT and ALP levels) and IgM levels at the time of PBC diagnosis before UDCA treatment. The correlation coefficients between fractalkine levels and ALT, ALP, and IgM levels were 0.12, 0.06, and 0.10, respectively, which were not significant. B: Correlation between serum fractalkine levels and autoantibodies at the time of PBC diagnosis before UDCA treatment. The correlation coefficients between fractalkine levels and levels of anti-centromere antibody, AMA, and gp210 antibody were 0.14, 0.18, and 0.001, respectively, which were not significant. However, a clear trend between fractalkine levels and the gp210 antibody titers was found; patients with high gp210 titers had low fractalkine levels, and patients with high fractalkine levels had low gp210 titers except one case (\#). This exceptional case (\#) had a high fractalkine level (33.2 ng/ml) and a high gp210 titer (132.4) and was clinically unique.

Fig. 3 Correlation between serum fractalkine levels and histological activation, stage, and three findings defining stage at the time of diagnosis of PBC before UDCA treatment. Serum fractalkine was significantly higher in cases with marked chronic cholangitis activity (CA3) than those with lower CA scores (CA0-CA2). In contrast, it was lower in cases with marked hepatitic activity (HA3) compared with those with lower HA scores (HA0-HA2). Among the three histological findings of fibrosis, bile duct loss, and Orcein-positive granules, the cases with low scores (0-1) for fibrosis, a score of 0 for Orcein-positive granules, and also early stages (stage 1-2) showed significantly higher fractalkine levels than those with a scores of 
2-3 for fibrosis and 1-3 for Orcein-positive granules, and advanced stages (stage 3-4). The statistical analysis was performed without the unique case (\#).

Fig. 4 Change in serum fractalkine levels before (before Tx) and 1 or 2 years after the beginning UDCA treatment (after Tx). "Total" is the data for all PBC cases. Among the three items defining the response to UDCA (serum ALP, ALT, and IgM levels), "good response, $\geq 2$ items" cases with high fractalkine levels $(>5 \mathrm{ng} / \mathrm{ml}$ ) before treatment showed a decrease after treatment, whereas “good response, $\leq 1$ item” cases showed an increase. 


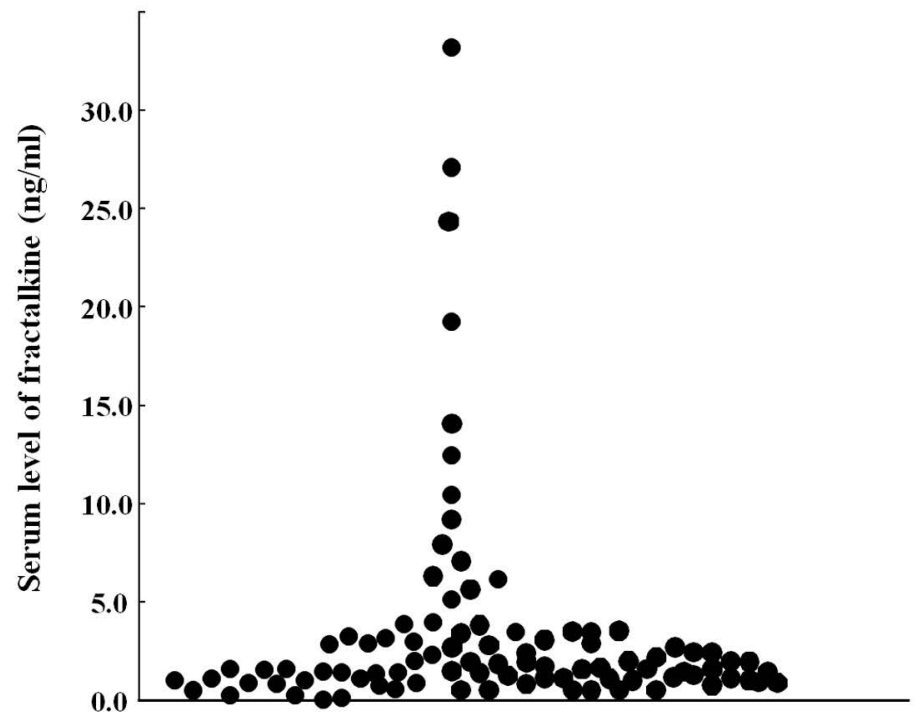

Fig.1 


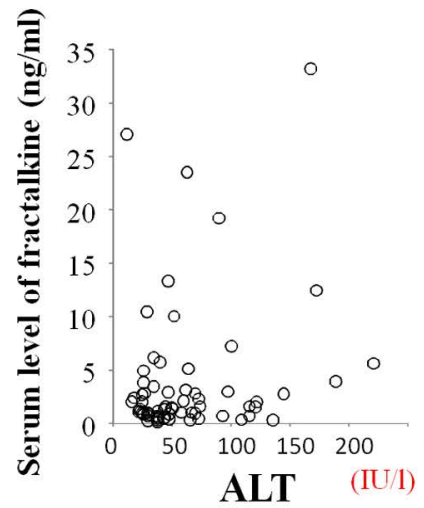

coefficient of correlation

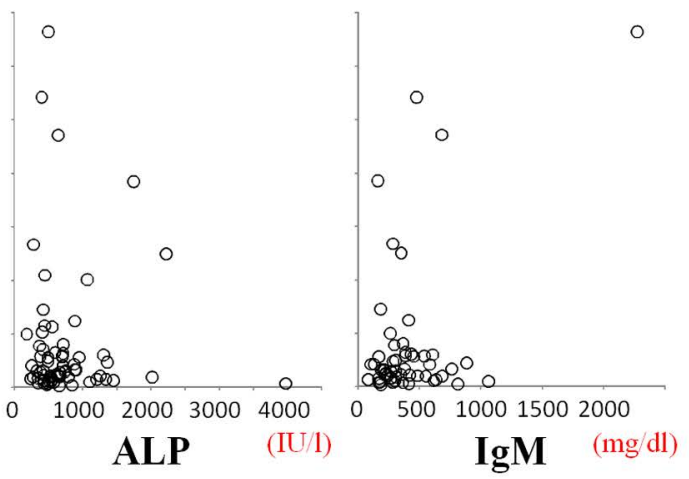

0.06

0.10

Fig.2A 


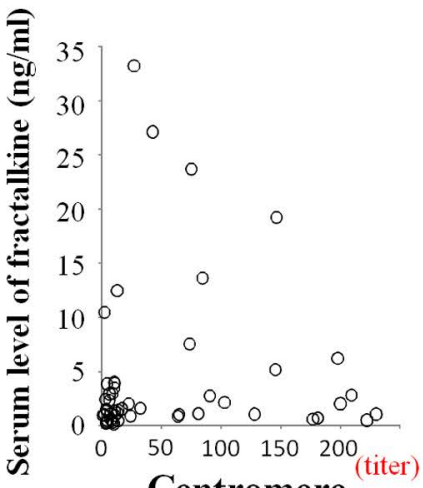

Centromere

coefficient of correlation

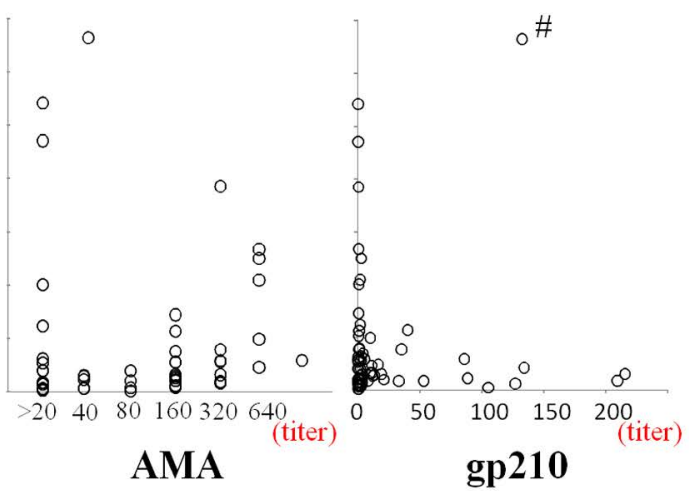

0.18

0.001

Fig.2B 

A Cholangitis activities
B Hepatitis activities
C stage
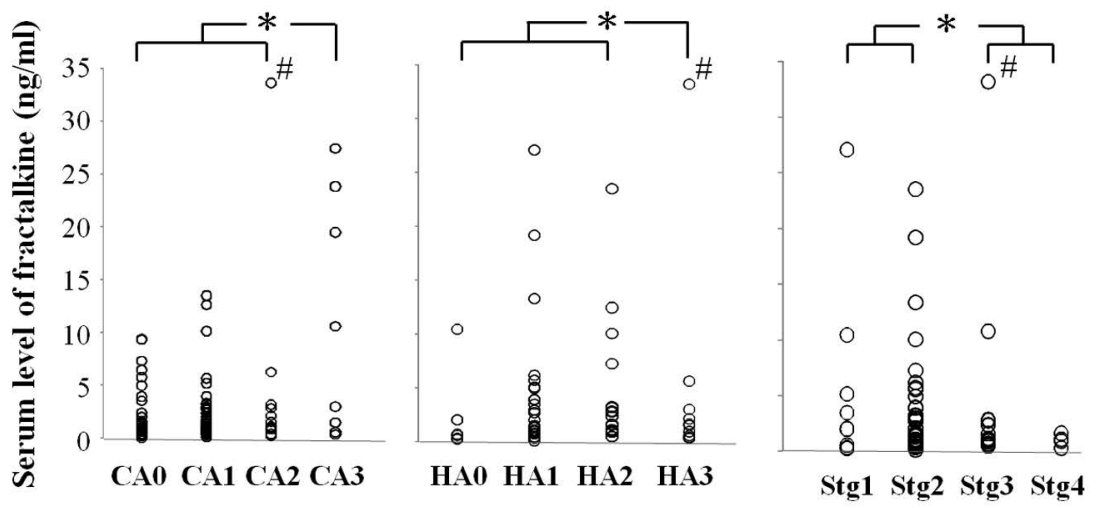

$(*, \mathbf{p}<0.05)$

Fig.3A-C 
D Fibrosis

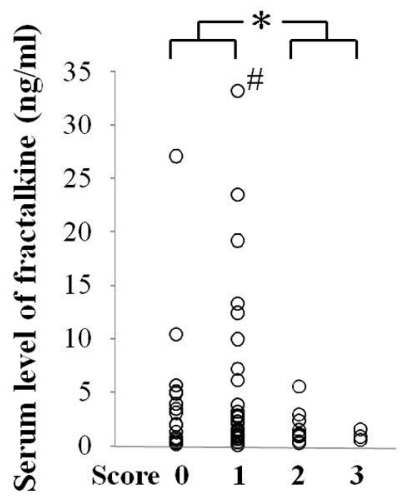

E Bile duct loss

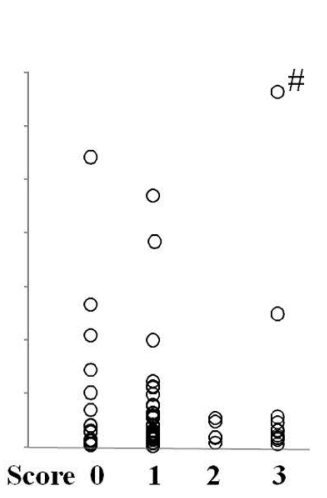

F Orcein-positive granule

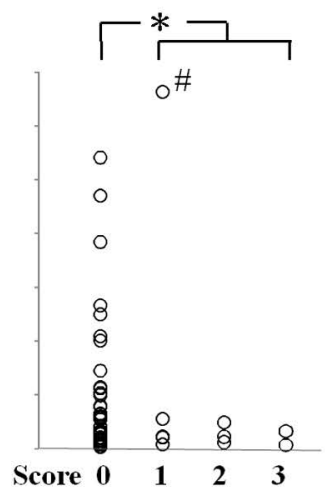

Fig.3D-F

$$
(*, \mathbf{p}<0.05)
$$



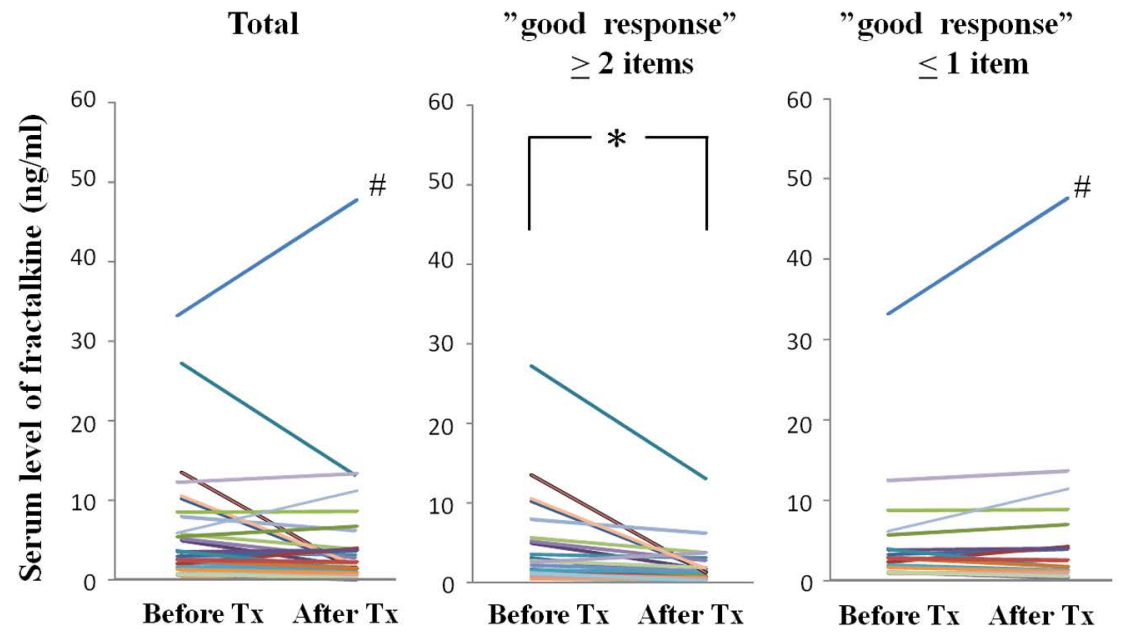

Fig.4 